\section{Compensation for Injury}

The Royal Commission on Civil Liability and Compensation for Personal Injury, who began by inviting evidence on injuries suffered in the course of employment and road transport injuries, are now extending their invitation to the remaining areas of their remit. These are: death or personal injury "through the manufacture, supply or use of goods or services; on premises belonging to or occupied by another; or otherwise through the act or omission of another where compensation under the present law is recoverable only on proof of fault or under the rules of strict liability."

Organizations or individuals wishing to submit evidence should send this to the Secretary, The Royal Commission on Civil Liability and Compensation for Personal Injury, 22 Kingsway, London WC2B 6LE. (Tel. 01-242 6828, Ext. 509.)

\section{British Association for Rheumatology and Rehabilitation}

At the annual general meeting held on 4 April the following officers were elected: president (1974-6), Dr. D. R. L. Newton; honorary treasurer (1974-7), Dr. R. Grahame; honorary secretary (1974-5), Dr. J. A. Mathews.

\section{COMING EVENTS}

Association of Police Surgeons of Great Britain.-Annual conference, 15-18 May, Newcastle upon Tyne. Details from the honorary Dudley, Worcs. DY1 2AW. (Tel. Dudley 52136).

Royal Society of Medicine.-Jephcott lecture, "Future Invention in a Harsher World" by Dr. A Spinks, 22 May, 6 p.m. Admission by ticket. obtainable from the information officer, $R$. S. M.,
1 Wimpole Street, London W1M 8AE, (Tei. 01-580 2070.)

Royal College of Surgeons of England.-College evening, "Pelvic Fractures and their Complications," 3 June. Fee $£ 2$ (including supper). Applications to the surgical training officer, WC2A 3PN. (Tel. 01-405 3474.)

Society for Research into Hydrocephalus and Spina Bifida.-Annual meeting. $13-15$ June, Gothenburg. Details from Dr. K. M. National School of Medicine, Health Park, Cardiff CF4 4XN. (Tel. 0222 755944, extn. 2287).

\section{SOCIETIES AND LECTURES}

For attending lectures marked a fee is charged or a ticket is required. Applications

\section{Monday, 6 May}

INSTITUTE OF LARYNGOLOGY AND OTOLOGY.-5.30 p.m., Clinicopathological conference.
RoYAL PoSTGRADUATB MEDICAL SCHOOL. -4.30 p.m., RoYAL PoSTGRADUATB MEDICAL SCHOOL. -4.30 p.m.,
Professor J. Scadding: Diffuse Pulmonary ROYAL SOCIBTY OF TROPICAL MEDICINE AND HYGIENE. - At Manson House, 26 Portland Place, Warrell, and Dr. P. Lewis: "Rabies in Zaria immunodiagnosis and pathophysiology.

\section{Tuesday, 7 May}

AdLerian Socirty of GraAt BritanN.-At Alliance Hall, Palmer Street, London S.W.1, 7.30 p.m., Dr. E. Weissmann: Overcoming Fear and Anxiety. D. H. W. Kelly: Investigation and Treatment of

\section{Wednesday, 8 May}

INSTITUTB OF Psychiatry.-5.30 p.m., Mr. P. H. Schurr: Psychosurgery.
ROYAL COLLEGE OF PHYSICIANS OF LONDON.- 5 p.m.,
Watson Smith lecture by Professor D. Watson Smith lecture by Professor D. J. of Haemoglobin Synthesis.

ROYAL COLlEGE OF SURgeons OF ENGLaND. -6 p.m., Hunterian lecture by Professor G. Bentley. Articular Cartilage Studies and Osteoarthritis.

ROYAL POSTGRADUATE MEDICAL SCHOOL. - 2 p.m., Dr. E. Wiltshaw: Non-Hodgkin's Lymphoma; tives in Occupational Pulmonary Disease.

\section{Thursday, 9 May}

ABERDEEN UNIVERSITY.-5 p.m., Marnoch lecture by Professor L. E. Gelin (Gothenburg): Kidney Transplantation as Method of Choice for Treatment of Terminal Renal Failure.

ONYMAN GILLESPIE LECTURE.-At Royal Infirmary, Edinburgh, 5 p.m., Dr. I. W. B. Grant: Byres,

Barley, Birds, and Breathlessness.

CYL COLLEGE OF PHYSICIANS OF LONDON.-5 p.m., pathic Oedema of by Dr.

Royal Postgraduate Medical School.-4.30 p.m. Dr. D. A. J. Tyrrell, F.R.S.: Chemotherapy of

ST. JoSEPH'S HOSPICE, LoNDON.-At Mare Street, Hackney, 8 p.m., Sister B. Saunders: Colostomy ST. MARY's HoSpital Medical SCHOOL. - 5.15 p.m., Dr. D. B. Scott: Effects of Inferior Vena Caval
Occlusion in Pregnancy.

\section{Friday, 10 May}

NUFFIELD DEPARTMENT OF SURGERY LECTURE THEATRE, RADCLIFFE INPIRMARY. -5 p.m. Litch-
field lecture: Professor Richard B. Goldbloom: field lecture: Profess

Paediatric Non-diseases.
Royal POSTGRADUATE MEdical SCHOOL.-11 a.m.,

ROYAL POSTGRADUATE MEDICAL SCHOOL.-11 a.m.,
Professor F. Fontan (Bordeaux): Surgical TreatProfessor F. Fontan (Borde

ROYAL CollEgE OF SURGEONS OF EDINBURGH.4.30 p.m., Mr. M. H. Irving: The Surgeon's
Contribution to the Management of Hodgkin's Disease.

\section{Saturday, 11 May}

Cambridge University Medical School.-At New Addenbrookes' Hospital, 10 a.m., day symposium: Family Planning.*

\section{UNIVERSITIES AND COLLEGES}

\section{ROYAL COLLEGE OF PHYSICIANS OF}

At a quarterly meeting of the college, held on 25 April, with the president, Sir Cyril Clarke, F.R.S., orary Fews:

Professor E. B. Ford, F.R.S., Professor Dorothy C. Hodgkin, O.M., F.R.S., Sir Peter Medawar, Whitteridge, Sir Frank Young, F.R.S.

Dr. D. A. Pyke was elected to serve as registrarThe following appointments were approved Dr. A. Herxheimer as college representative on the Medico-Pharmaceutical Forum.

Dr. A. Hollman as college representative on the committee of management of the Chelsea Physic Garden. C. S. Nicol, Dr. D. H. G. MacQuaide and Dr. C. S. Nicol, Dr. D. H. G. MacQuaide and
Dr. P. A. J. Ball as college representative on the Dr. P. A. J. Ball as college representa

Dr. J. P. Bull as college representative on the Dr. J. Apley to serve as a member of the M.R.C.P. part II question group.

Dr. P. R. Evans to serve as a member of the
Joint Committee on Psychological Medicine to repre-

Dr. $R$. Goulding to serve as a member of the
sent paediatrics. Fluoridation Committee as a toxicologist.

The following were elected Fellows:

F. Arden, Margaret I. Griffiths, Enid I. M Addenbrooke, P. J. Sweeney, H. J. W. Ficher, Monica K. McAllen, L. R. Patel R. C. S. Dick, Abrahams, V. H. Allan, R. C. Angove, P. J. A.
Blaney, T. G. Fox, M. F. Hart, L. W. Carstairs, R. G. Dreadon, F. W. B. H. Hurlburt, Vivien U. Lutwyche, E. J. T. Pretteiohn, F. L. Ritchie. G. F
Swann, A. W. Branwood, Maduravasal V. Chari, Swann, A. W. Branwood, Maduravasal V. Chari,
W. B. D. Maile. G. H. Wattlev, A. El-S. Eissa, Pach, Fox, R. Ke V. Hart W. M. Irwin, P. G Keates, A. J. Barnett. F. A. Binks. S. H. C. Wright, $R$. J. Young, L. Capner, D Collins, I. W. L. Edwards T. E. Parry, R. Se:de'in S '
Steel. P. F. Bartlev, P. R. Swyer, G. Howells, Steel. P. F. Bartley, P. R. Swyer, G. Howells, Constanre M. Hecling. W. L. Sanders, R H. Vines,
H. E. Parry. L. Vngelooel, G. S. Nagy. T. H.
Renwick. S. E T. Rnhertson. K. Simnoon. I K.

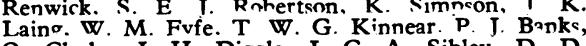
O Ciarke. J. H. Digole. J C . A S Shev D D. Felix-Davies D M. W. M. Vine, Invre B Burke,
M. H. Watson, W. K. Stewart, R. S. Thanabalasundrum, John Angus, B. A. Bradlow, H. Conen, F. G. Paxon, I. E. Evans, R. G. Gibbs, J. Knox, M. D. Turner, E. Wilkes, N. Cardoe, A. A. Glynn, Apthorp, F. D. Kitchin, Madeleine B. Morris, D. W. Davies, M. Sandler, A. Hordern, F. L. Elphick, E. A. Nieman, N. J. W Wallooppillai, H. Barkley, C. McCance, John Yell, T. B. Begg, M. P. H. Davies, K. H. Nickol, M. M. S. Ahuja, C. P. Aber, Margaret H. J. Weston, R. E. Eban, Maguire, E. D. Sever, L. Wollner, J. H. Baron,
I. B. Munro, M. J. Grayson, B. E. Kendall, J. T.
Smyth, D. A. H. Yates, W. Hindle, I. B. Houston, M. L. Thomas, R. S. Kocen, D. W. Evans, O. W. Hill, J. L. Reed, A. K. Thould, I. V. Wilson, Montgomery, S. Posen, J. R. Smythies, D. W. R. Sumner, J. R. Tighe, Patricia G. Wallis, M. F. Waters, E. M. P. Brett, D. A. Chamberlain, J. B. L. Gee, D. R. K. Medley, N. B. Pride, R. C. Richards, McCarthy, B. H. B. Robinson, B. A. Scobie, I. Weinbren, W. B. Hanley, J. M. Parkin, J. J. S. Comaish, H. M. Hodkinson, L. Stimmler, Junro, B. M. Phillips, M. J. Purves, R. Summerly, G. S. Thompson, L. Sinclair, P. Stephenson, P. S. W. Wilkins, D. Barltrop, E. R. Beck, W. O. McCormick, A. J. Ralston, J. A. P. Trafford, D. H. A. Robinson, Barbara J. Boucher, M. S. Gotsman, M. W. Greaves, C. D. Holdsworth, R. Brown, K. Horsfield, D. Hull, F. J. C. Millard, Pauline S. Monro, J. A. Wilson, A. C. Graham, R. E. Kendell, J. Meecham, C. G. Miller, Eileen N. Thompson, J. Rhodes, L. Horwich, R. E. Nagle, B. L. Pimstone, J. A. Tulloch, K. W. Heaton, R. Smith, Breckenridge, B. O. Osuntokun, S. R. Meadow, B. A. Walker, T. J. Ryan, S. W. Clarke, H. Stott,
H. Watson, J. Hoenig. H.. C. Burry, T. C. N. Gibbens, P. A. B. Raffle, H. Baderman, R. Harris, Zaimis, Cicely M. S. Saunders, G. H. Tovey, J. D. Whiteside, I. D. P. Wootton. R. J. Linden, Rosemary P. Biggs, J. A. Dudgeon, P. K. Bondy, L. J. Bruce-Chwatt, Sir John Gray, F.R.S. G. A. Lindeboom, v. A. McKusick, D. M. Mitchell, B. A. Rexed, A. S. Wiener, K. J. R. Wightman.
The following were elected and admitted as members: Adelstein, R. E. C. Altounvan, J. Dobbing, Peckham, Joan Slack, Sheila M. Worrledge.

\section{Corrections}

\section{Haemophilus influenzae}

We regret that owing to a printing error the letter from Drs. K. Cartwright and D. C. Turk (27 April, p. 225) was given an incomplete heading. This should have read "Haemophilus influenzae Cellulitis."

\section{Studies on Gastrointestinal Cells}

The Handbook and Atlas of Gastrointestinal Exfoliative Cytologv by Joáo Carlos Prolla and Joseph B. Kirsner (6 April, p. 64) is published by M.I.T. Press as stated.

\section{Notice to Authors}

When original articles and letters for publication are not submitted exclusively to the British Medical fournal this must be stated. Acrepted articles may subsequently be selected for publication in the North American monthly edition.

Correspondence on editorial business should be addressed to the Editor, British Medical fournal, B.M.A. House, Tavistock Square, London WC1H 9JR. Telephone: 01-387 4499. Telegrams: Aitiology, London, W.C.1.

Authors wanting reprints of their articles should notify the Publishing Manager, B.M.A. House, Tavistock Square, WC1H 9JR, on receipt of proofs.

\section{(C) British Medical Journal 1974}

All Rights Reserved. No part of this publication may be reproduced, stored in a retrieval system, or transmitted, in any form or by any means, electronic, mechanical, photocopying, recording or otherwise, without the prior permission of the British Medical Fournal 